\title{
Laparoscopic Partial Nephrectomy
}

\author{
Sandeep Gurram, MD and Louis Kavoussi, MD
}

\begin{abstract}
Renal cell carcinoma has historically been managed by radical nephrectomy, but as knowledge of the disease has advanced, nephron sparing surgery has become the norm in appropriately selected tumors. Laparoscopic partial nephrectomy (LPN) has been shown to have comparable oncologic outcomes and a shorter convalescence period compared to the traditional open partial nephrectomy. Dissemination of techniques has led to significantly increased use of minimally invasive nephron sparing surgery, and LPN remains a cost-effective and efficacious method for approaching small renal masses.
\end{abstract}

Keywords: laparoscopy, nephrectomy, renal cancer, minimally invasive surgical procedures, laparoscopic partial nephrectomy

\section{Indications}

A BSOLUTE INDICATIONS FOR laparoscopic partial nephrectomy (LPN) include bilateral tumors or tumors in a solitary kidney. Relative indications include familial renal cancer syndromes such as Von Hippel-Lindau, hereditary leiomyomatosis, or hereditary papillary renal cell carcinoma (RCC). Patients with chronic kidney disease are generally offered nephron sparing surgery for hope of future renal function preservation. This reasoning also applies to those patients with preexisting diseases that may threaten a solitary kidney such as uncontrolled diabetes and hypertension. The American Urologic Association renal mass guidelines extend this rationale to other threatened populations such as those with recurrent urolithiasis or morbid obesity. ${ }^{1}$ The current standard of care is to perform a partial nephrectomy for pT1a tumors assuming that the mass is amenable to such an approach. Although originally reserved for exophytic and small $(<4 \mathrm{~cm})$ tumors, multiple series have shown that LPN can be safely offered for hilar, endophytic, and multiple tumors. ${ }^{2-4}$

Relative contraindications to LPN include tumor thrombi into the renal vein or inferior vena cava or advanced tumor invasion, as these are best approached with a radical nephrectomy. Uncorrected coagulopathy also is a relative contraindication to LPN.

\section{Preoperative Preparation}

The preoperative work-up for a patient with a renal mass starts with a detailed history and physical examination. Laboratory analysis should include a complete blood count, basic metabolic profile, calcium, alkaline phosphatase, and a coagulation panel. Radiographic evaluation requires cross- sectional imaging of the abdomen with contrast provided that the patient is able to receive contrast. Assessing the location and complexity of the mass and mapping the number and course of the renal vessels are important for preoperative planning. A chest X-ray or CT is necessary to screen for pulmonary metastasis. A bone scan is unnecessary in the absence of bone pain or elevated alkaline phosphatase, and a head CT is also not required unless neurologic changes are evident. The presence of gross hematuria or hilar tumors is suggestive of urothelial cell carcinoma, and delayed contrast imaging with excretory phases can help differentiate the two malignancies.

We routinely use a mechanical bowel preparation the day before surgery; however, many surgeons choose to forgo this step. The patient is kept on a clear liquid diet the day before surgery. They are given eight ounces of magnesium citrate midday on the day before surgery to help with evacuation of the bowels.

\section{Patient Positioning}

The operating room is configured to maximize ease of operability and visibility to all involved in the procedure. As is standard, the anesthesiologist is at the head of the bed, while the scrub nurse is situated opposite the surgeon. Most of the instruments are located on a separate table at the foot of the bed, while the most actively used instruments are placed on a Mayo stand above the feet of the patient or in the pockets of the surgical drape itself. For the transperitoneal approach, the assistant generally stands on the same side as the surgeon until it is time to extract the specimen at which point they will move to the opposite side. Monitors are placed on each side

The Smith Institute for Urology, Donald and Barbara Zucker School of Medicine at Hofstra/Northwell, New Hyde Park, New York, USA. 
of the patient to allow for both the surgeon and the scrub nurse to view the operation.

For a transperitoneal partial nephrectomy, the patient is placed in a modified flank position. A gel dome positioner is placed vertically behind the shoulders to create a $45^{\circ}$ angled position. The lower arm is placed on an arm board, and the upper arm is slightly bent and draped over the chest to gently lie on top of the chest and lower arm (Fig. 1). The bed is not flexed as it is generally not needed to help gain exposure. The upper arm elbow is padded, and the patient is secured to the table with generous amounts of 3 inch silk tape across the chest and hips. A light amount of tape is used to secure the legs and upper arm to prevent them from shifting. The bed is lowered and will be rotated toward the surgeon after port placement.

\section{Surgical Steps}

\section{Trocar placement}

The Veress needle is generally placed at the umbilicus. If there is concern for significant amount of adhesions at this location, use a site at least $4 \mathrm{~cm}$ under the costal margin at the midclavicular line on the side to be operated on. Observing the initial pressure once insufflation has started is important to ensure appropriate placement. Initial patient pressure less than $10 \mathrm{~mm} \mathrm{Hg}$ is consistent with appropriate peritoneal placement of the Veress needle. Higher pressures may indicate occlusion of the tip of the needle by viscera so the abdominal wall is lifted and the needle is turned anteriorly. If high pressures persist, the needle is removed completely and reinserted.

Local anesthetic is administered, and a skin incision is made to accommodate a $10 \mathrm{~mm}$ screw trocar just lateral to the umbilicus. The laparoscope is inserted, and the abdomen is inspected for injury. Two additional trocars are placed, a $5 \mathrm{~mm}$ subxiphoid working port and an ipsilateral $12 \mathrm{~mm}$ lateral working port $\sim 2$ to $3 \mathrm{~cm}$ superior and medial to the anterior superior iliac spine. An optional fourth $12 \mathrm{~mm}$ working trocar can also be placed 2 to $3 \mathrm{~cm}$ superior to the pubic symphysis (Fig. 2). This port is generally converted to the specimen extraction site when in use to improve cosmesis and to lower the chance of postoperative incisional hernias. Trocars may need to be shifted laterally to accommodate for more obese patients.

\section{Transperitoneal LPN}

\section{Reflection of colon}

Once ports are placed, the surgery is started by incising the white line of Toldt. In this study, we prefer to use the laparoscopic DeBakey forceps and monopolar cautery shears. It is important to be in the correct plane for this portion of the procedure. The incision is made just lateral to the colonic mesentery but not excessively lateral as that will start mobilizing the lateral aspect of the kidney much too early (Fig. 3). The depth of the incision should be enough to enter the peritoneum at which point the colon and the mesentery can be swept medially with blunt dissection. Switching to blunt dissection here prevents inadvertent early entry into Gerota's fascia and subsequent loss of appropriate planes of dissection. This incision is taken caudally to the level of the bifurcation of the iliac vessels. Once the peritoneum is incised the relatively avascular plane is entered, and the colon will sweep easily. The mesentery is identified by its golden yellow hue, and medial pressure should be applied at the interface between the mesenteric fat and Gerota's fascia to sweep the colon and mesentery (Fig. 3). Any areas of remaining attachment can be cauterized with the monopolar shears and dissected free to prevent tearing of any vessels.

\section{Splenic mobilization}

For left sided procedures, the incision on the white line is carried superiorly and then medially around the splenic flexure. The colon is then continued to be bluntly dissected off of Gerota's fascia. Blunt dissection can be facilitated best with the $5 \mathrm{~mm}$ irrigator-aspirator or as we prefer with a $10 \mathrm{~mm}$ LigaSure (Valleylab, Boulder, CO) bipolar sealer/ divider. Once the colon is swept away, the splenic attachments
FIG. 1. Modified flank positioning before tilting the table.

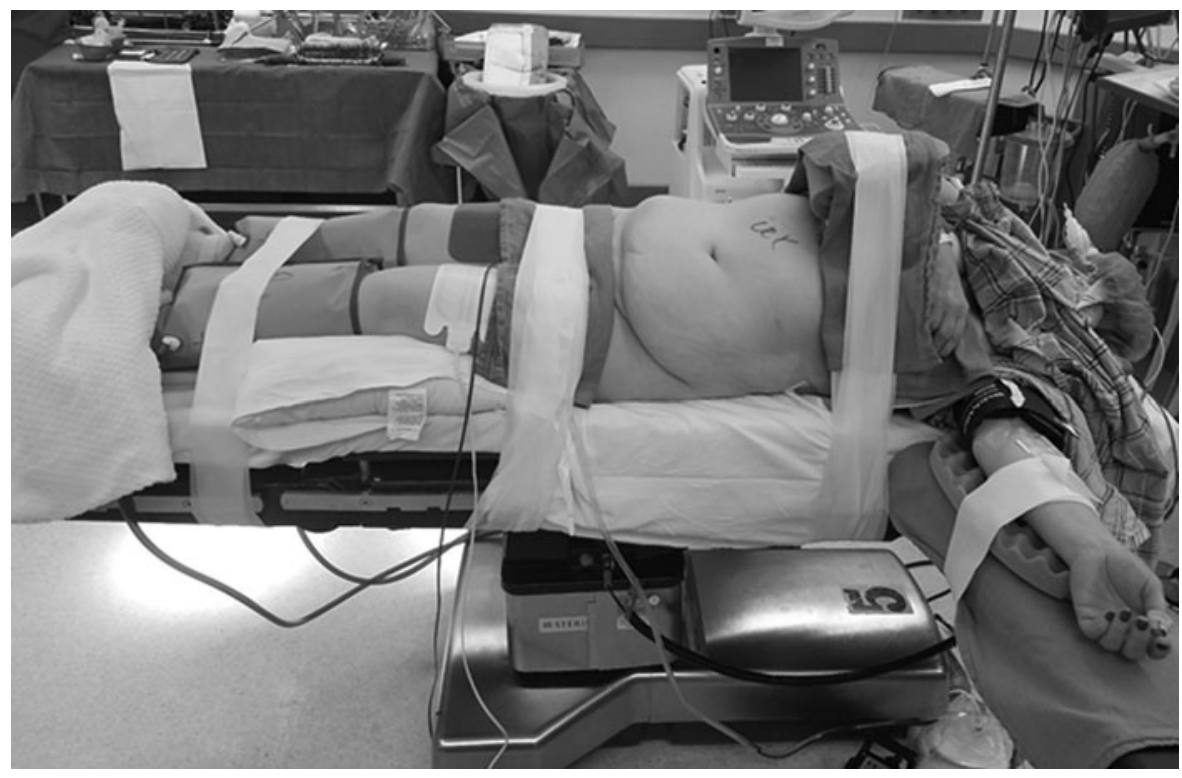




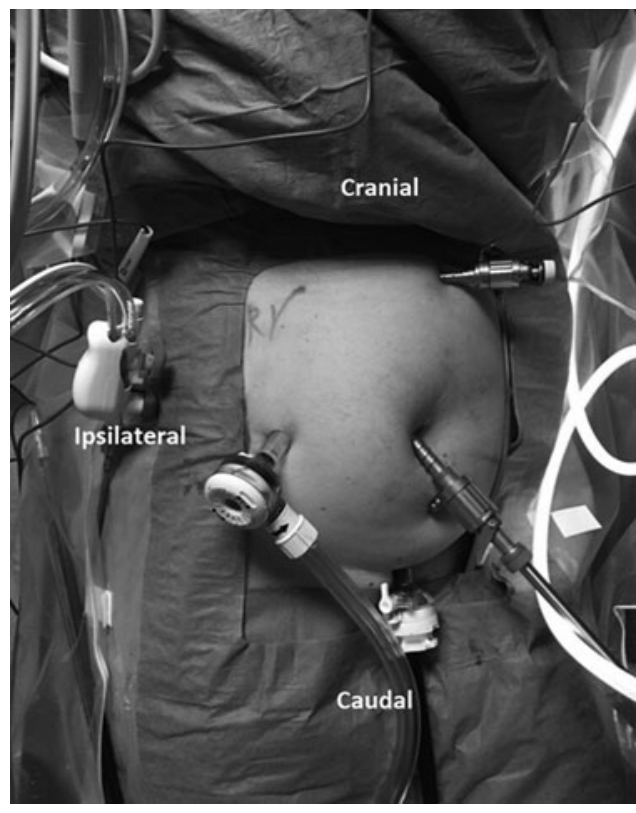

FIG. 2. Port placement. Insufflation port is lateral, while in a nonobese patient the rest of the ports are placed in the midline.

can be easily visualized. The lateral splenorenal ligament is ligated with monopolar cautery or the bipolar sealer. Care is taken to dissect toward the spleen when dissecting free these ligaments. Excessive tension on any ligament attached to the spleen causes capsular tearing and considerable bleeding. If this is encountered, argon electrocautery can be helpful in controlling bleeding. Once the splenorenal ligament is divided, the spleen can start to be mobilized medially. At this point the splenocolic ligament can be approached and divided in a similar manner. This will allow the spleen and pancreas to be mobilized medially en bloc. During this maneuver, the splenic flexure and descending colon can fall into the operative field. The use of a paddle retractor can help with exposure and keeping medial tension on the tissue.

\section{Right sided mobilization}

For right sided procedures, the first step is to mobilize the liver. Unlike the spleen, the liver is more mobile and less

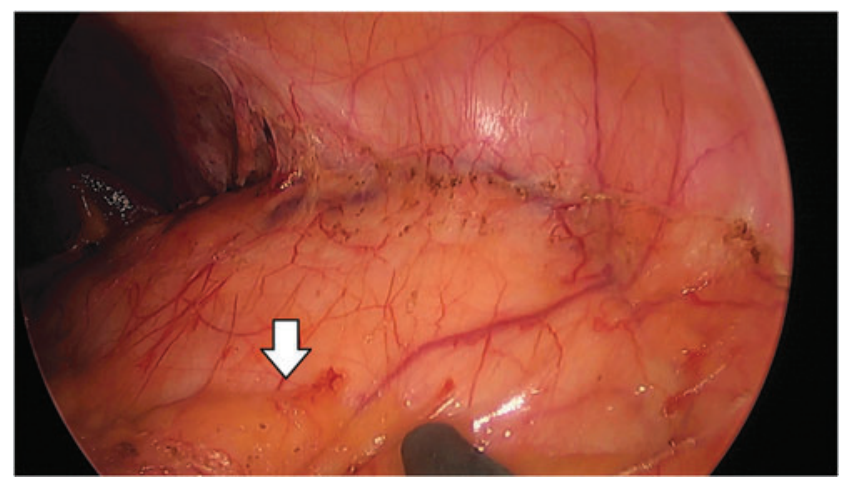

FIG. 3. Arrow shows the location of the colonic mesentery. prone to traction injury or capsular tearing. Using monopolar shears or a bipolar sealer, the lateral attachments of the liver to the side wall are dissected free. The liver is placed on some gentle upward tension from an instrument placed in the upper most trocar, and any hepatorenal ligaments are divided with either hook electrocautery or monopolar shears. Once the liver is mobilized, the colon is then dissected free and mobilized medially as described in the prior segment. The ascending colon and hepatic flexure lie on the anterior surface of the kidney and may be more medially located than the descending colon or splenic flexure, and therefore, excessive lateral dissection should be avoided. Once the colon has been mobilized, the duodenum will be visualized. Kocherization is performed athermally with Endo Shears.

\section{Identifying the ureter}

The psoas muscle can be a useful landmark in identifying the mid ureter. The ureter will generally be found medial to the psoas and deep to the gonadal vein. With the use of a suction-aspirator and DeBakey forceps, the inferior pole of the kidney is lifted. Using blunt dissection, either instrument is advanced deeper until the abdominal side wall is reached. The kidney is then aggressively lifted, allowing better visualization and later mobilization of the ureter and the hilum. Once appropriate tension has been placed, the plane underneath the kidney is developed. It is best to use a blunt $10 \mathrm{~mm}$ instrument at this point to help sweep away tissue to create a working space and allow for even more upward tension to be placed on the kidney. Next, the fat and connective tissue is dissected free, and the gonadal vein and ureter can be located. The ureter is elevated, while the gonadal vein is kept free from tension (Fig. 4).

\section{Hilar dissection}

With the kidney lifted anterolaterally, the hilum and the medial attachments of the kidney are placed on tension. With the use of the aspirator-irrigator and electrosurgical shears, the dissection is carried cranially until the hilum is identified. The renal vein is generally identified first and frequently an anterior veil of overlying adventitial tissue can be present on top of it (Fig. 5). This tissue is best approached by removing the anterior-lateral tension on the kidney and grasping the tissue with the laparoscopic DeBakey forceps. This veil or sheet of tissue is gently dissected medially off of the hilum and taken with bipolar cautery. The kidney is once again placed on anterolateral tension, and the perihilar dissection is started. This tissue can contain small vessels that can be easily sheared, and therefore, care is taken to appropriately cauterize or clip and ligate any that are visualized. Be wary of excessive clip usage should the need rise for possible conversion to nephrectomy as these clips can interfere with possible future staple lines. The irrigator-aspirator can also be used to gently tease off the fibrofatty tissue overlying the renal vein, but excessive tension should be avoided to prevent a venotomy or shearing of any vessels overlying the vein.

The renal artery is dissected in a similar manner. If an anterior view to approach the artery is unsuccessful to adequately localize it, the camera can be advanced lateral to the hilum and the $30^{\circ}$ scope angled toward the hilum for a posterior view. Although some prefer skeletonization of the vessels individually, the hilum can be clamped en bloc. 


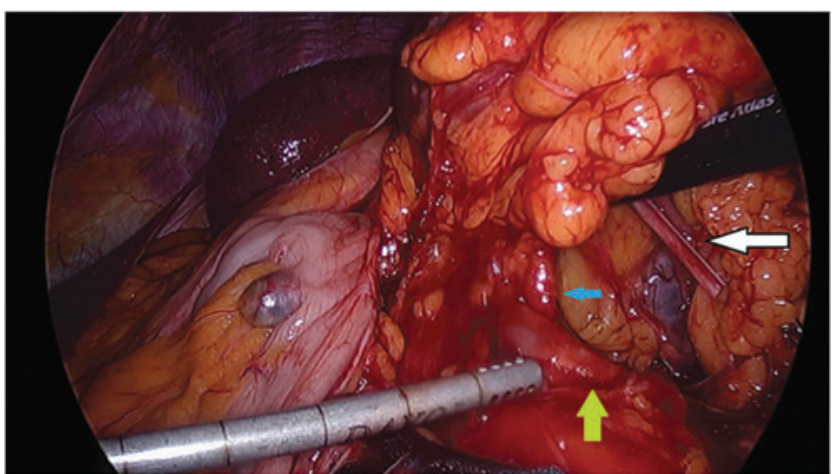

FIG. 4. Left kidney lifted anteriorly and placed on tension. White arrow: ureter. Green arrow: gonadal vein. Blue arrow: renal artery.

Once the inferior aspect of the renal hilum is cleared, the superior plane is developed. This will allow for appropriate placement of laparoscopic bulldog clamps around the hilum (Fig. 6). The kidney is once again tented up anteriorly, but it is important to ensure that the tension is placed closer to the renal hilum as opposed to the lower pole of the kidney. Dissection is then started slightly superior to the cranial edge of the renal hilum. Once again, a combination of the laparoscopic DeBakey and irrigator-aspirator can be used to sweep away a small amount of fibrofatty tissue in this location. Once this small space has been created, a blunt $10 \mathrm{~mm}$ instrument can be used to develop the space enough so that the jaws of the laparoscopic bulldog clamps can be placed around the hilum. Care is taken in this area on the left sided tumor to prevent inadvertent damage to the adrenal vein.

\section{Lateral and superior dissection}

The kidney will generally need to be mobilized unless facing an anteriorly located tumor. To do so, the lower pole of the kidney is placed on upward tension. The ureter and gonadal vein are identified and swept down medially and are purposefully excluded from the anterior tension. The entirety of the lateral dissection is performed with a curved sweeping motion and can be expedited with a $10 \mathrm{~mm}$ LigaSure. The curved sweeping motion will allow for the plane to be de-

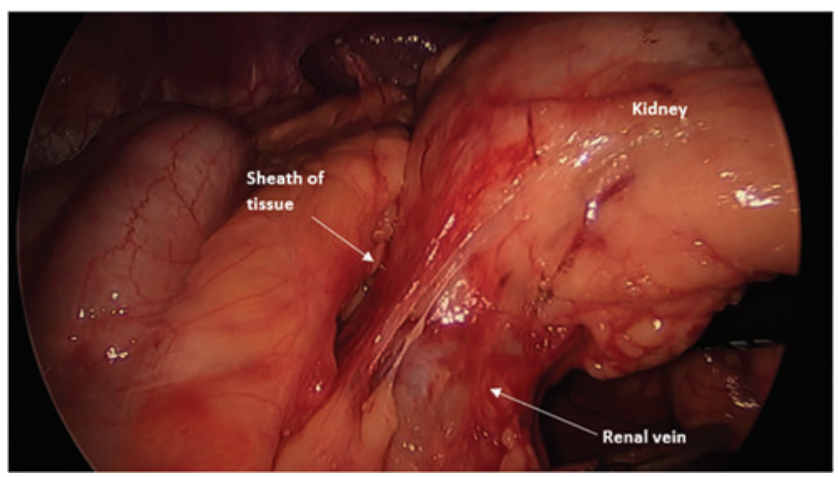

FIG. 5. Kidney lifted anterio-laterally causing the hilum to be placed on tension. The anterior sheath of tissue overlying the renal vein is identified and ligated.

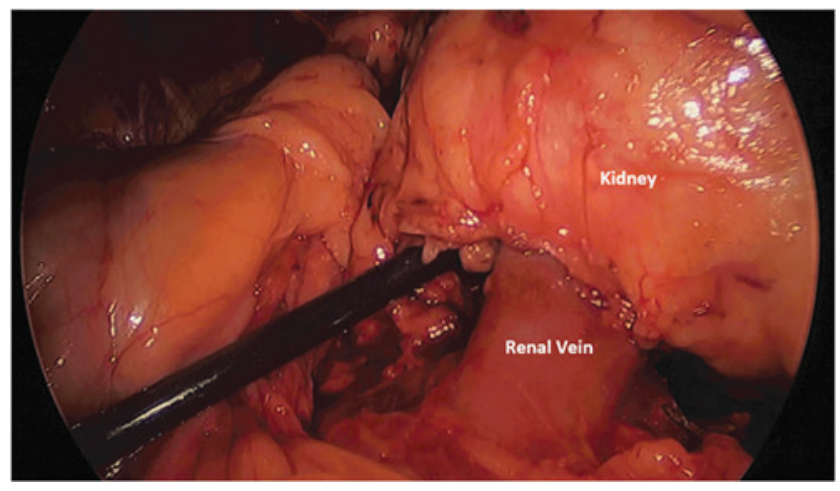

FIG. 6. The space superior to the renal vein is developed to allow for placement of a vascular clamp over the hilum.

veloped bluntly at which point the tissue can be ligated. It is important to keep the perinephric fat with the kidney while performing the dissection. This dissection is continued superiorly until the kidney is fully mobilized.

\section{Tumor excision}

Once the kidney has been appropriately mobilized, intraoperative ultrasonography is performed with a laparoscopic transducer to identify the tumor. The edges of the tumor are noted, and the perinephric fat is entered slightly away from the site. This dissection is taken down to the renal capsule. In this study, the aspirator-irrigator can be helpful in dissecting the fat to the level of the renal capsule. A circumferential cuff of fat is dissected free from the renal mass leaving a cap of fat overlying the mass. Understanding that most renal masses are generally spherical in configuration will help guide the direction of the dissection and help prevent any inadvertent entries into the tumor. The edge of the tumor is then scored circumferentially with a hook electrocautery or Endo Shears (Fig. 7). If considering dissecting the tumor off with parenchymal fracturing technique, then the renal capsule should also be entered and scored with $5 \mathrm{~mm}$ hook electrocautery.

After the tumor has been scored, laparoscopic bulldogs are placed on the renal hilum. Generally two are placed on both the renal artery and vein if clamping en bloc; otherwise, the renal artery is clamped individually first and is then followed by the vein. If fracturing the tissue, the aspirator-irrigator is used to dissect on the site that has been previously scored (Fig. 8). The pressure on the renal parenchyma will lead to tearing and fracturing of the parenchyma on the prior scored plane. As vessels are encountered, a laparoscopic right angle dissector is used to clear the tissue behind and around the vessel. The vessel is then ligated with a Hem-o-lok clip (Weck Closure Systems, Triangle Park, NC) and divided (Fig. 9). This is performed circumferentially until the tumor has been dissected free. Another method of dissecting the tumor is with the use of cold Endo Shears to excise the tumor along the same plane. It is important not to excise the mass with cautery as the char will discolor the dissection plane and make it difficult to differentiate normal renal parenchyma from tumor. The same method of isolating and ligating vessels should be used. 


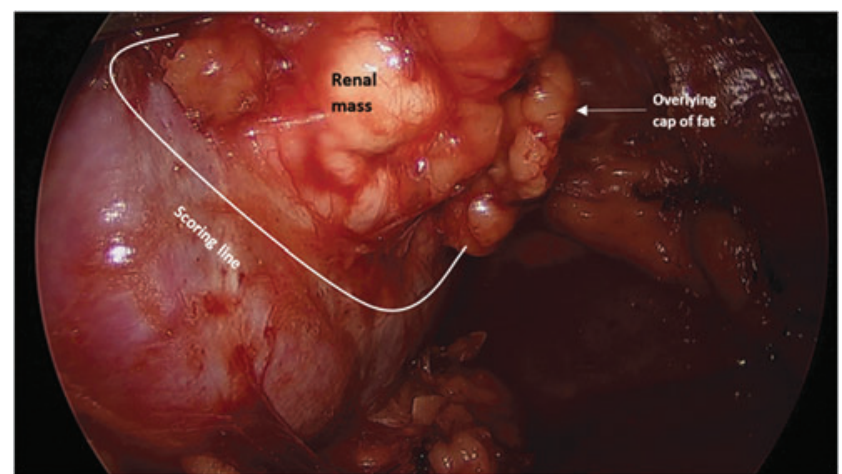

FIG. 7. The renal mass is cleared of adjacent fat, and the base is scored with hook electrocautery.

Care is taken not to travel too wide on the dissection as excessive amount of normal renal parenchyma can be removed inadvertently. This is most common during the second half of the tumor dissection as the prior scored segment can be more difficult to visualize. During the dissection it is important to use appropriate tension and counter-traction on the tissue. This can be done by grasping the fat overlying the tumor; however, care should be taken not to pull it off of the tumor. As the plane is developed more, DeBakey forceps can be used to grasp the base of the tumor and apply appropriate counter-traction without removing the perinephric fat.

Once excision is complete, a tumor base specimen can be sent for frozen section pathologic analysis although modern studies have questioned the utility of obtaining a frozen section. The base of the tumor is then coagulated with argon electrocautery. Argon electrocautery should not be performed on the or near the collecting system. Traditional maxim states that collecting system defects are repaired with a two-layer closure technique. Our experience, however, has shown that single layer mass closure is as effective as a two layer closure in repairing both the parenchymal and collecting system defects and reduces warm ischemia time (WIT). Closure is performed with a running mattress suture with 2-0 V-Loc barbed suture (Covidien, Norwalk, CT). The suture should be cinched after each full pass of the suture

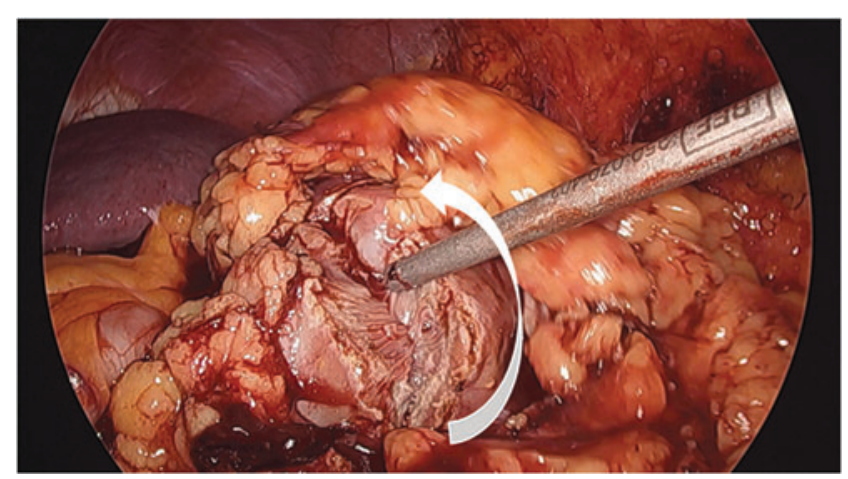

FIG. 8. Once scored, the parenchyma is fractured with use of the irrigator-aspirator. Gentle force should be applied perpendicular to the fracture line as is demonstrated by the white arrow. through the parenchyma. It is important that the hand furthest away from the parenchyma holds the needle in the same direction and plane as the suture was thrown when cinching. Otherwise, holding the suture on tension in any other angle can lead to tearing of the parenchyma (Fig. 10). After the final throw, the end of the suture can be secured with a Hem-o-lok clip.

The specimen is placed in an EndoCatch bag (Auto Suture, Norwalk, CT) and removed through the suprapubic port site. A Carter-Thomason closure device is used under direct visualization to close the lateral trocar site with a 0 Vicryl suture. A drain may be placed if concerned for possible collecting system involvement. It is placed underneath the ureter and is brought out of the abdomen laterally. At this point, the gas should be evacuated from the abdomen, and all incisions closed in a subcuticular manner.

\section{Postoperative Care}

Patients are started on a clear liquid diet postoperatively. The Foley catheter is removed on the first postoperative day, and the diet is advanced once they have return of gastrointestinal function. Patients are strongly encouraged to ambulate and participate in incentive spirometry. If a drain was placed, then before discharge a fluid creatinine is checked to ensure that there is no urine extravasation before removal of the drain.

\section{Troubleshooting}

Obese patients represent a population that uniquely benefits from the laparoscopic approach. Compared to open surgery, we have shown that obese patients have decreased operative time, blood loss, WIT, complications, and hospital stay when undergoing LPN. ${ }^{5}$ LPN has also been shown to have similar perioperative outcomes between normal and obese patients. ${ }^{6}$ When operating on obese patients, it is important to note that the trocars may need to be laterally shifted to avoid having difficulties with instruments reaching the desired location. Morbidly obese patients may benefit from bariatric trocars; however, generally this is not necessary.

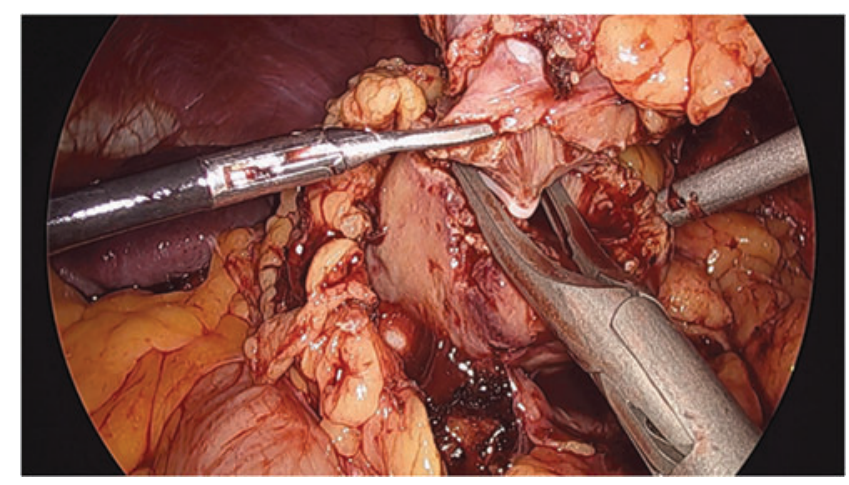

FIG. 9. Hem-o-lok clips are used on any vessels that are encountered. It is important to dissect around the tissue to create a packet of tissue that can be easily incorporated by the clip. 
FIG. 10. It is important when cinching the suture to place tension in the same direction as the suture was thrown (white arrow). This will prevent parenchymal and capsular tears.

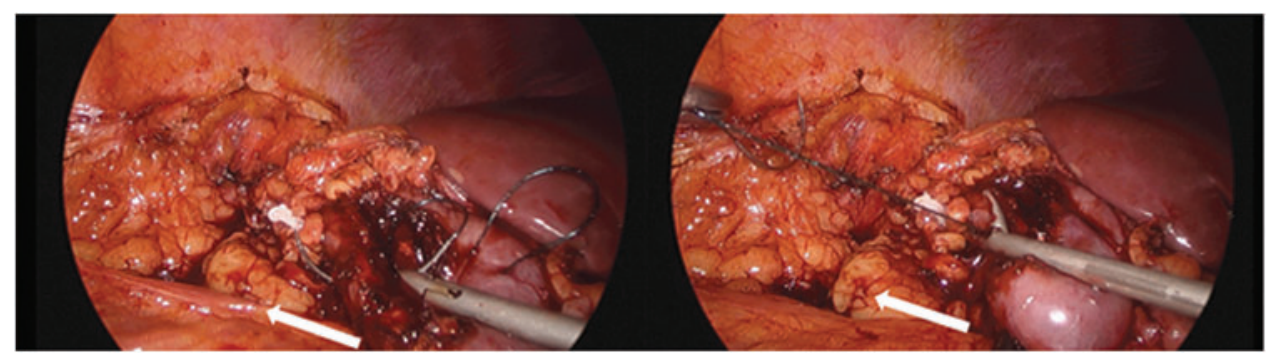

Multiple vessels or anomalous renal vasculature can also add to the complexity of an LPN. Cross-sectional imaging is crucial for preoperative planning and delineation of the renal vasculature. LPN has been shown to be as safe in patients with multiple vessels as those with a single renal artery and vein and has not been shown to have differences in blood loss, WIT, operative time, or complications. ${ }^{7}$ Patients with multiple renal arteries can be amenable to selective clamping of vessels. ${ }^{8}$ Although polar vessels are easier to identify the segment of the kidney they supply, more central vessels may be more difficult to isolate for which region of the kidney they supply. Various methods are used to assess the renal segment supplied by each artery. The original method is simply observing which portion of the kidney had visual ischemic changes after clamping a single artery. ${ }^{9}$ Others have used near-infrared fluorescence imaging with indocyanine green to help identify which artery should be clamped to allow for a zero-ischemia LPN. ${ }^{10}$

Hereditary renal cancer syndromes such as Von HippelLindau offer a unique set of challenges. Generally debulking surgery is started once a renal mass has reached $3 \mathrm{~cm}$ as rates of metastasis are extremely low in tumors smaller than this. ${ }^{11}$ To preserve as much renal function as possible, some surgeons have preferred performing a tumor excision or enucleation without clamping the renal hilum. In addition, given the likelihood of needing to reoperate on the kidney, Gerota's fascia is replaced and reapproximated to reduce adhesions.

\section{Alternate Approaches}

There are several situations such as multiple intraperitoneal surgeries or prior intraperitoneal approaches to an ipsilateral renal mass in which the ability to perform a retroperitoneal LPN is quite useful. The retroperitoneal approach to the kidney is described elsewhere in this atlas, but the renal mass excision is still the same. The renal hilum is clamped, and excision is performed by scoring the renal capsule and excising the mass. The absence of space with retroperitoneoscopy can be challenging, and appropriate finger dissection and balloon dissection are important to create an appropriate working space. ${ }^{12}$

\section{Outcomes}

Minimally invasive partial nephrectomy is becoming the standard of care for most small renal masses. This prevailing shift in the evidence has been endorsed by many national urologic and cancer associations. ${ }^{1,13}$ Multiple studies have shown that minimally invasive partial nephrectomies do not have worse complications, WIT, positive margins, or oncologic outcomes compared to open partial nephrectomies, and they offer faster convalescence and decreased postoperative pain. ${ }^{14,15}$

Earlier comparative studies between open and laparoscopic partial nephrectomies examined heterogeneous patient populations with differing tumor sizes and unclear complexity. These studies generally showed that LPN was most often performed on smaller tumors, had longer ischemia time, and higher complication rates, but overall operative time, estimated blood loss, and length of stay were considerably less. ${ }^{16-18}$ As LPN became more prevalent, newer studies with matched cohort analyses demonstrated that once renal mass size and complexity were matched, there were considerable benefits with LPN. LPN showed decreased ischemia time, operative time, length of stay, and blood loss (see Table 1). ${ }^{19,20}$

In the early years of laparoscopic surgery, LPN was the predominant minimally invasive approach to treating the small renal mass. Over time, the further dissemination and increased availability of the robotic platform started resulting in increased use of robotic partial nephrectomies (RPNs). This trend eventually led to increased adoption of RPN, which subsequently surpassed the rate of LPN use. Survey of the 2008 to 2010 Nationwide Inpatient Sample Database showed that of the 38,064 partial nephrectomies that were performed, $23.9 \%$ of patients underwent RPN compared to 9.2\% who underwent LPN. ${ }^{21}$ Increasing utilization of RPN was seen over time in the database with an increase from $23.9 \%$ to $49 \%$ by $2012 .^{22}$ It should be noted that these changes were not borne from improvements seen in patient driven quality outcomes from RPN, as high quality, wellcontrolled data unfortunately do not exist comparing LPN to RPN. $^{23}$ The most comprehensive retrospective analysis comparing LPN to RPN demonstrated no major differences in complications, operative time, serum creatinine, estimated blood loss, or positive margin rate. ${ }^{24}$ The study did note a significantly lower rate of conversion to open or conversion to radical nephrectomy and a decrease in WIT and change in glomerular filtration rate (GFR). However, even this study had considerable issues such as multiple duplicated patient cohorts, inadequate follow-up for LPN, its retrospective nature, and inclusion of inconsequential clinical outcomes such as a mean improvement of $4.5 \%$ in estimated GFR across studies. ${ }^{23}$ The comparative ease of performing a RPN, which has been demonstrated in the steep learning curve of LPN, has been an oft-cited reason to adopt the technique. ${ }^{25}$ However, it is important for surgeons to use a discriminating eye to ensure that patient outcomes, not technology, should drive treatment options. 


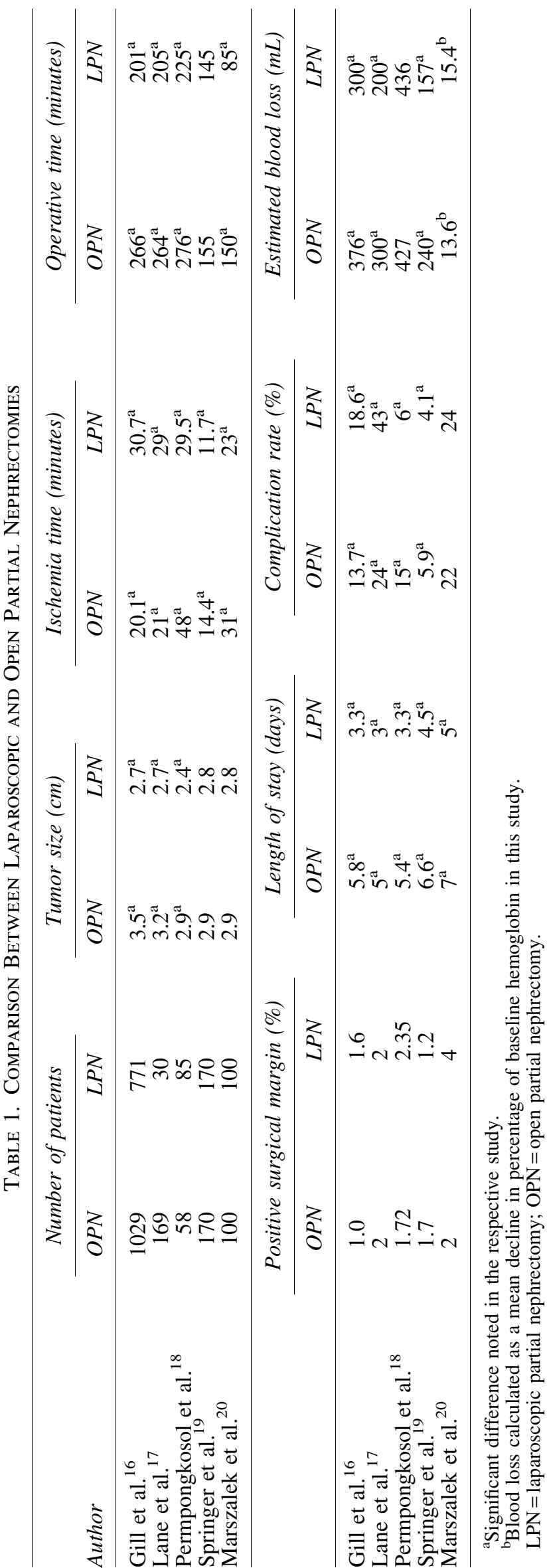

\section{Recommended Videos from Videourology}

1. Videourology 2018, Vol. 32, No. 2

Robot-Assisted Laparoscopic Partial Nephrectomy of Hilar Renal Tumor

Ronit Patnaik, Cheuk Fan Shum, and Chandru P. Sundaram

https://www.liebertpub.com/doi/10.1089/vid.2017.0028

2. Videourology 2016, Vol. 30, No. 6

Feasibility of Omitting Cortical Renorrhaphy During Robot-Assisted Partial Nephrectomy

Andrew Vissing, Steven Kheyfets, Clinton Bahler, and Chandru Sundaram

https://www.liebertpub.com/doi/full/10.1089/vid.2016.0041

3. Videourology 2017, Vol. 31, No. 4

Evolution of Technique from Minimally Ischemic to Pure Off-Clamp Partial Nephrectomy: 15-Year Learning Curve and Outcomes of 1083 Patients

Giuseppe Simone, Gabriele Tuderti, Leonardo Misuraca, Mariaconsiglia Ferriero, Francesco Minisola, Giuseppe Romeo, Manuela Costantini, Salvatore Guaglianone, and Michele Gallucci

https://www.liebertpub.com/doi/full/10.1089/vid.2017 .0005

4. Videourology 2011, Vol. 25, No. 1

Robot-Assisted Partial Nephrectomy*

Shyam Sukumar, Quoc-Dien Trinh, Firas Petros, and Craig Roger

*A full-length, coordinating article is available in Journal of Endourology 25/2 at www.liebertonline.com/end/doi/ full/10.1089/end.2010.0672

https://www.liebertpub.com/doi/full/10.1089/vid.2010 .0117

5. Videourology 2011, Vol. 25, No. 1

Laparoscopic Partial Nephrectomy: A Decade of Evolution*

Andre K. Berger, Robert J. Stein, Inderbir S. Gill, and Mihir M. Desai

*A full-length, coordinating article is available in Journal of Endourology 25/2 at www.liebertonline.com/end/doi/ full/10.1089/end.2010.0676

https://www.liebertpub.com/doi/full/10.1089/vid.2010 .0156

\section{Author Disclosure Statement}

No competing financial interests exist.

\section{Funding Information}

No funding was received for this article. 


\section{Supplementary Data}

Supplementary Video S1 Full

Supplementary Video Step 1

Supplementary Video Step 2

Supplementary Video Step 3

Supplementary Video Step 4

\section{References}

1. Campbell S, Uzzo RG, Allaf ME, et al. Renal mass and localized renal cancer: AUA guideline. J Urol 2017;198: 520-529.

2. George AK, Herati AS, Rais-Bahrami S, Waingankar N, Kavoussi LR. Laparoscopic partial nephrectomy for hilar tumors: oncologic and renal functional outcomes. Urology 2014;83:111-115.

3. Shikanov S, Lifshitz DA, Deklaj T, Katz MH, Shalhav AL. Laparoscopic partial nephrectomy for technically challenging tumours. BJU Int 2010;106:91-94.

4. Abreu AL, Berger AK, Aron M, et al. Minimally invasive partial nephrectomy for single versus multiple renal tumors. J Urol 2013;189:462-467.

5. Romero FR, Rais-Bahrami S, Muntener M, Brito FA, Jarrett TW, Kavoussi LR. Laparoscopic partial nephrectomy in obese and non-obese patients: Comparison with open surgery. Urology 2008;71:806-809.

6. George AK, Rothwax JT, Herati AS, et al. Perioperative outcomes of laparoscopic partial nephrectomy stratified by body mass index. J Endourol 2015;29:1011-1017.

7. Singh D, Finelli A, Rubinstein M, Desai MM, Kaouk J, Gill IS. Laparoscopic partial nephrectomy in the presence of multiple renal arteries. Urology 2007;69:444-447; discussion 447.

8. Shao P, Tang L, Li P, et al. Precise segmental renal artery clamping under the guidance of dual-source computed tomography angiography during laparoscopic partial nephrectomy. Eur Urol 2012;62:1001-1008.

9. Shao P, Qin C, Yin C, et al. Laparoscopic partial nephrectomy with segmental renal artery clamping: Technique and clinical outcomes. Eur Urol 2011;59:849-855.

10. Lee HJ, Chen K, Molchanov R, Schwentner C, Sim ASP. Feasibility of utilizing near-infrared fluorescence imaging with indocyanine green for super-selective arterial clamping in pure laparoscopic partial nephrectomy. Int $\mathbf{J}$ Urol 2018;25:382-383.

11. Duffey BG, Choyke PL, Glenn G, et al. The relationship between renal tumor size and metastases in patients with von Hippel-Lindau disease. J Urol 2004;172:63-65.

12. Matsuda $T$, Nakagawa $M$, Oguchi $N$, et al. Retroperitoneoscopic partial nephrectomy with transient occlusion of renal artery for treatment of small renal tumors. Urology 2004;64:26-30.

13. Ljungberg B, Bensalah K, Canfield S, et al. EAU guidelines on renal cell carcinoma: 2014 update. Eur Urol 2015;67: 913-924.

14. Lane BR, Campbell SC, Gill IS. 10-year oncologic outcomes after laparoscopic and open partial nephrectomy. J Urol 2013;190:44-49.

15. Minervini A, Siena G, Antonelli A, et al. Open versus laparoscopic partial nephrectomy for clinical T1a renal masses: A matched-pair comparison of 280 patients with
TRIFECTA outcomes (RECORd Project). World J Urol 2014;32:257-263.

16. Gill IS, Kavoussi LR, Lane BR, et al. Comparison of 1,800 laparoscopic and open partial nephrectomies for single renal tumors. J Urol 2007;178:41-46.

17. Lane BR, Novick AC, Babineau D, Fergany AF, Kaouk JH, Gill IS. Comparison of laparoscopic and open partial nephrectomy for tumor in a solitary kidney. J Urol 2008;179: 847-851; discussion 852.

18. Permpongkosol S, Bagga HS, Romero FR, Sroka M, Jarrett TW, Kavoussi LR. Laparoscopic versus open partial nephrectomy for the treatment of pathological T1N0M0 renal cell carcinoma: A 5-year survival rate. J Urol 2006;176: 1984-1988; discussion 1988-1989.

19. Springer C, Hoda MR, Fajkovic H, et al. Laparoscopic vs open partial nephrectomy for T1 renal tumours: Evaluation of long-term oncological and functional outcomes in 340 patients. BJU Int 2013;111:281-288.

20. Marszalek M, Meixl H, Polajnar M, Rauchenwald M, Jeschke K, Madersbacher S. Laparoscopic and open partial nephrectomy: A matched-pair comparison of 200 patients. Eur Urol 2009;55:1171-1178.

21. Ghani KR, Sukumar S, Sammon JD, Rogers CG, Trinh QD, Menon M. Practice patterns and outcomes of open and minimally invasive partial nephrectomy since the introduction of robotic partial nephrectomy: Results from the nationwide inpatient sample. J Urol 2014;191:907-912.

22. Bahler CD, Monn MF, Flack CK, Gramm AR, Gardner TA, Sundaram CP. Assessing cost of robotic utilization in partial nephrectomy with increasing utilization. J Endourol 2018;32:710-716.

23. George AK, Hartman C, Kavoussi LR. Robotic partial nephrectomy: The Will Rogers surgical effect. Eur Urol 2016;69:7-8.

24. Choi JE, You JH, Kim DK, Rha KH, Lee SH. Comparison of perioperative outcomes between robotic and laparoscopic partial nephrectomy: A systematic review and metaanalysis. Eur Urol 2015;67:891-901.

25. Potretzke AM, Bhayani SB. Laparoscopic partial nephrectomy: Rest in peace. Eur Urol 2015;67:902-903.

Address correspondence to: Sandeep Gurram, MD

The Smith Institute for Urology Donald and Barbara Zucker School of Medicine at Hofstra/Northwell 450 Lakeville Road New Hyde Park, NY 11042 USA

E-mail: sandeep.gurram@nih.gov

\begin{aligned} & \multicolumn{1}{c|}{ Abbreviations Used } \\ $\mathrm{CT} & =$ computed tomography \\ $\mathrm{GFR} & =$ glomerular filtration rate \\ $\mathrm{LPN} & =$ laparoscopic partial nephrectomy \\ $\mathrm{OPN} & =$ open partial nephrectomy \\ $\mathrm{RPN} & =$ robotic partial nephrectomy \\ $\mathrm{WIT} & =$ warm ischemia time \end{aligned}

